\title{
Percutaneous Needle Biopsy in the Diagnosis of Liver Diseases in Children
}

\author{
SeyedMohsen Dehghani ${ }^{1,{ }^{*},}$, Mahmood Haghighat ${ }^{2}$, MohammadHadi Imanieh ${ }^{1}$, Bita Geramizadeh ${ }^{1}$, \\ Zahra Eskandari ${ }^{2}$,Fatemeh Erfanifar ${ }^{2}$,Abdorrasoul Malekpour ${ }^{2}$ \\ ${ }^{1}$ Shiraz Transplant Research Center, School of Medicine, Shiraz University of Medical Sciences, Shiraz, IR Iran \\ ${ }^{2}$ Gastroenterohepatology Research Center, Nemazee Teaching Hospital, School of Medicine, Shiraz University of Medical Sciences, Shiraz, IR Iran \\ *Corresponding author: Seyed Mohsen Dehghani, Gastroenterohepatology Research Center, Shiraz Transplant Research Center, Nemazee Teaching Hospital, School of Medicine, \\ Shiraz University of Medical Sciences, Shiraz, IR Iran. Tel: +98-7116125849, Fax: +98-7116474298, E-mail: dehghanism@sums.ac.ir.
}

Received: May 16, 2013; Revised: July 20, 2013; Accepted: July 24, 2013

Background: Liver is prone to different diseases including metabolic, congenital, infections, drug injuries, and malignancies. Liver biopsy is an aggressive but definitive way to diagnose liver diseases.

Objectives: The present study is designed to evaluate the results of liver biopsies in children referred to the Pediatric Gastroenterology Department of Nemazee Hospital.

Materials and Methods: The present retrospective study was conducted on 308 liver biopsy specimens from children suspected to liver diseases who had been referred to Pediatric Gastroenterology Department affiliated to Shiraz University of Medical Sciences between March 2003 and March 2008.

Results: Totally 308 liver biopsies from the children aged less than 18 years was investigated. There were 128 girls and 180 boys with mean age of $6.04 \pm 5.97$ years and Male/Female ratio of 1.4/1. The most common pathologic findings were chronic hepatitis $(n=71 ; 23.1 \%)$ metabolic diseases $(n=37 ; 12.1 \%)$, cirrhosis $(n=27 ; 8.8 \%)$, secondary hemochromatosis $(n=22 ; 7.1 \%)$, neonatal hepatitis $(n=22 ; 7.1 \%)$, and hepatic malignancies ( $n=21 ; 6.8 \%$. Liver biopsy was non diagnostic in $16.2 \%(n=50)$ of the patients.

Conclusions: Chronic hepatitis, metabolic liver diseases, cirrhosis, and neonatal hepatitis are the most common liver diseases in children in our center. In this study a high percentage of liver biopsies were non-diagnostic. Therefore, providing the necessary facilities and equipment for special assessment of liver tissue are essential in this center.

Keywords: Biopsy; Liver Diseases; Children

\section{Background}

Liver biopsy is an important procedure in the diagnosis and follow-up of liver diseases in children, while invasive, enjoys a relatively low major complication rate. Pediatric liver diseases include a broad spectrum of disorders such as developmental abnormalities, metabolic and infectious disorders that finally result in liver cirrhosis and failure. Hepatic disorders are one of the most significant causes of morbidity and mortality in children $(1,2)$. Treatment strategies are different for each disorder; so, it is obvious that a correct and prompt diagnosis has an important role in proper management of these children. For example, during early infancy, the clinical presentations of cholestasis caused by neonatal hepatitis and biliary atresia are very similar and the correct and definite diagnosis might be very difficult. Considering this fact that biliary atresia needs surgical intervention as soon as possible, in order to get a better outcome, a correct and prompt diagnosis is very important. Histopathological examination of the liver biopsy is the individual most reliable test for the differential diagnosis of infants and children who are suspected to liver diseases $(2,3)$.

\section{Objectives}

The aim of this study was to evaluate the spectrum of liver diseases diagnosed by percutaneous liver biopsy in children of our referral center.

\section{Materials and Methods}

In this retrospective cross-sectional study, all the liver biopsy specimens taken from children with suspected liver disease aged less than 18 years between March 2003 and March 2008 who had been admitted in Pediatric Gastroenterology Department of Nemazee Hospital affiliated to Shiraz University of Medical Sciences were reviewed. During this five-year period, a total of 329 percutaneous liver biopsies were obtained in Pediatric Gastroenterology Department of Nemazee Hospital. All liver

Implication for health policy/practice/research/medical education:

Liver biopsy is an aggressive but definitive way to diagnose the liver diseases. The present study has investigated the results of liver biopsies in children and reported the most common pathologic findings in this group of patients.

Copyright (c) 2013, Iranian Society of Pediatrics. This is an Open Access article distributed under the terms of the Creative Commons Attribution License (http:/ creativecommons.org/licenses/by/3.0), which permits unrestricted use, distribution, and reproduction in any medium, provided the original work is properly cited. 
biopsies were done by pediatric gastroenterology fellows who were trained for this procedure. Abdominal ultrasonography and coagulation study was performed in all patients before obtaining the liver biopsies. In patients with coagulopathy or thrombocytopenia, percutaneous liver biopsy was performed only after elimination of the coagulopathy or thrombocytopenia. The written consent of the patients' parents was obtained by explaining the need for liver biopsy and its possible side effects. Closed biopsy of the liver through the skin was performed using Gallini No. 16 and 18 needles (Italy). Patients were not allowed to eat four hours before and after the biopsy. During this period, sugar-electrolyte containing fluids and calories were supplied to control body water and also vital signs were monitored serially by a physician after the sampling. All of the liver samples were placed in formalin solution (10X) in a volume of 20 times more than the biopsy size for histopathologic studies. All specimens were sent to Pathology Department of Nemazee Hospital for histopathologic evaluation. Of these, 21 specimens were excluded either due to insufficient liver tissues (less than three portal tracts) or no submitted liver tissue at all. A total of 308 liver biopsies taken from 128 girls and 180 boys suspected to have liver diseases were evaluated and reported by an experienced hepatopathologist. Afterwards, the frequency of each disease was measured and recorded according to the age and sex of the patients. Also the pattern of liver diseases was evaluated in different age groups; less than two $(n=123 ; 39.9 \%), 2$ - $6(n=32$; $10.4 \%), 6-12(n=59 ; 19.2 \%)$, and $12-18$ years $(n=94 ; 30.5 \%)$.

\section{Results}

Totally, 308 liver biopsy specimens consisting 128 girls (41.6\%) and 180 boys (58.4\%) with a mean age of $6.04 \pm$ 5.97 years were reviewed. The most common histopathological diagnosis in children was chronic hepatitis $(\mathrm{n}=$ 71; 23.1\%) followed by metabolic disorders including hereditary tyrosinemia type 1 , glycogen and lipid storage diseases $(n=37 ; 12.1 \%)$, cirrhosis $(n=27 ; 8.8 \%)$, secondary hemochromatosis due to major thalassemia ( $n=22 ; 7.1 \%)$, neonatal hepatitis ( $n=22 ; 7.1 \%$ ), hepatic malignancies including hepatoblastoma, hepatocellular carcinoma and lymphoma ( $\mathrm{n}=21 ; 6.8 \%)$, biliary atresia $(\mathrm{n}=14 ; 4.5 \%)$, and familial intrahepatic cholestasis ( $n=7 ; 2.3 \%$ ). Other less frequent diagnoses are presented in Table 1.

\begin{tabular}{llll}
\hline \multicolumn{1}{l}{ Table 1. Results of Liver Biopsies in Children Less Than 18 Years of Age in Different Sex Groups } & \multicolumn{1}{l}{ Total, No. (\%) } \\
\hline Disease & Female, No. (\%) & Male, No. (\%) & $71(23.1)$ \\
\hline Chronic hepatitis & $31(43.7)$ & $40(56.3)$ & $50(16.2)$ \\
\hline Non-diagnostic & $21(42)$ & $29(58)$ & $37(12.1)$ \\
\hline Metabolic diseases & $16(43.2)$ & $21(56.8)$ & $27(8.8)$ \\
\hline Cirrhosis & $12(44.4)$ & $15(55.6)$ & $22(7.1)$ \\
\hline Hemochromatosis & $6(27.3)$ & $16(72.7)$ & $22(7.1)$ \\
\hline Neonatal hepatitis & $7(31.8)$ & $15(68.2)$ & $21(6.8)$ \\
\hline Hepatic malignancy & $8(38.1)$ & $13(61.9)$ & $14(4.5)$ \\
\hline Biliary atresia & $5(35.7)$ & $9(64.3)$ & $13(4.2)$ \\
\hline Steatosis & $7(53.8)$ & $6(46.1)$ & $7(2.3)$ \\
\hline Familial intrahepatic cholestasis & $3(42.9)$ & $4(57.1)$ & $7(2.3)$ \\
Acute cellular rejection & $3(42.9)$ & $4(57.1)$ & $6(1.9)$ \\
Chronic granulomatous inflammation & $3(50)$ & $3(50)$ & $6(1.9)$ \\
\hline Hydatid cyst & $4(66.7)$ & $2(33.3)$ & $3(0.9)$ \\
\hline Paucity of intrahepatic bile ducts & $1(33.3)$ & $2(66.7)$ & $1(0.3)$ \\
\hline Eosinophilic hepatitis & $1(100)$ & - & $1(0.3)$ \\
\hline CMV hepatitis & - & $1(100)$ & 308 \\
\hline Total & 128 & 180 & \\
\hline
\end{tabular}

Liver biopsy was not diagnostic in $16.2 \%(n=50)$ of the patients. As shown in the Table 2 the most common liver diseases in children aged less than two years were metabolic disorders $(n=28 ; 22.8 \%)$ that included lipid storage disease $(n=11)$, glycogen storage disease $(n=9)$ and hereditary tyrosinemia type $1(n=8)$. Other common hepatic diseases in this age group were neonatal hepatitis
( $n=22 ; 17.9 \%)$, biliary atresia $(n=12 ; 9.7 \%)$, steatosis $(n=$ $12 ; 9.7 \%)$, hepatic malignancies mainly hepatoblastoma ( $\mathrm{n}$ $=11 ; 8.9 \%$ ), and cirrhosis $(n=9 ; 7.3 \%)$. The most common diagnosed liver diseases in children aged between two and six years were chronic hepatitis $(n=8 ; 25 \%)$ and metabolic disorders $(n=5 ; 15.6 \%)$ that included glycogen storage disease $(n=2)$, hereditary tyrosinemia type $1(n=2)$, 
and lipid storage disease $(\mathrm{n}=1)$. Other causes of liver disease in this age group of children are presented in Table 3. Chronic hepatitis ( $n=18 ; 30.5 \%)$, cirrhosis ( $n=8 ; 13.5 \%$ ), and secondary hemochromatosis due to major thalassemia $(n=7 ; 11.9 \%$ ) were the most common hepatic diseases among children aged between 6 and 12 years (Table 4). Table 5 shows the pattern of liver diseases in children between 12 and 18 years. Chronic hepatitis ( $\mathrm{n}=45 ; 47.9 \%$ ), secondary hemochromatosis due to major thalassemia ( $n=12 ; 12.8 \%)$, cirrhosis ( $n=9 ; 9.6 \%)$, and hepatic malignancies mainly hepatocellular carcinoma $(n=5 ; 5.3 \%)$ were the most common pathological findings in this age group of children.

Table 2. Results of Liver Biopsies in Children Aged Less Than two Years

\begin{tabular}{ll}
\hline Disease & No. (\%) \\
\hline Metabolic diseases & $28(22.8)$ \\
\hline Neonatal hepatitis & $22(17.9)$ \\
\hline Non-diagnostic & $20(16.3)$ \\
\hline Biliary atresia & $12(9.7)$ \\
\hline Steatosis & $12(9.7)$ \\
\hline Hepatic malignancy & $11(8.9)$ \\
\hline Cirrhosis & $9(7.3)$ \\
\hline Chronic granulomatous inflammation & $4(3.2)$ \\
\hline Familial intrahepatic cholestasis & $2(1.6)$ \\
\hline Paucity of intrahepatic bile ducts & $2(1.6)$ \\
\hline CMV hepatitis & $1(0.8)$ \\
\hline Total & $123(100)$ \\
\hline
\end{tabular}

Table 3. Results of Liver Biopsies in Children Aged Between two and six Years

\begin{tabular}{ll}
\hline Disease & No. (\%) \\
\hline Chronic hepatitis & $8(25)$ \\
\hline Non-diagnostic & $5(15.6)$ \\
\hline Metabolic diseases & $5(15.6)$ \\
\hline Hemochromatosis & $3(9.4)$ \\
\hline Familial intrahepatic cholestasis & $3(9.4)$ \\
\hline Hepatic malignancy & $2(6.2)$ \\
\hline Biliary atresia & $2(6.2)$ \\
\hline Cirrhosis & $1(3.1)$ \\
\hline Chronic granulomatous inflammation & $1(3.1)$ \\
\hline Paucity of intrahepatic bile ducts & $1(3.1)$ \\
\hline Acute cellular rejection & $1(3.1)$ \\
\hline Total & $32(100)$ \\
\hline
\end{tabular}

Table 4. Results of Liver Biopsies in Children Aged Between six and 12 Years

\begin{tabular}{ll}
\hline Disease & No. (\%) \\
\hline Chronic hepatitis & $18(30.5)$ \\
\hline Non-diagnostic & $11(18.6)$ \\
\hline Cirrhosis & $8(13.5)$ \\
\hline Hemochromatosis & $7(11.9)$ \\
\hline Hydatid cyst & $3(5.1)$ \\
\hline Hepatic malignancy & $3(5.1)$ \\
\hline Metabolic diseases & $3(5.1)$ \\
\hline Familial intrahepatic cholestasis & $2(3.4)$ \\
\hline Acute cellular rejection & $2(3.4)$ \\
\hline Chronic granulomatous inflammation & $1(1.7)$ \\
\hline Eosinophilic hepatitis & $1(1.7)$ \\
\hline Total & $59(100)$ \\
\hline
\end{tabular}

Table 5. Results of Liver Biopsies in Children Aged Between 12 and 18 Years

\begin{tabular}{ll}
\hline Disease & No. $(\%)$ \\
\hline Chronic hepatitis & $45(47.9)$ \\
\hline Non-diagnostic & $14(14.9)$ \\
\hline Hemochromatosis & $12(12.8)$ \\
\hline Cirrhosis & $9(9.6)$ \\
\hline Hepatic malignancy & $5(5.3)$ \\
\hline Acute cellular rejection & $4(4.2)$ \\
\hline Hydatid cyst & $3(3.2)$ \\
\hline Metabolic diseases & $1(1.1)$ \\
\hline Steatosis & $1(1.1)$ \\
\hline Total & $94(100)$ \\
\hline
\end{tabular}

Of 308 patients who underwent liver biopsy, 11 cases developed severe pain at the site of biopsy that needed narcotics and six cases complicated with subcapsular hematoma presenting with hypotension and pallor, five of them were resolved with conservative management and one case needed surgical repair. Also one patient complained of melena that improved with supportive care.

\section{Discussion}

Liver biopsy, though an invasive procedure, plays a vital role in the precise diagnosis in cases of hepatosplenomegaly, cholestatic jaundice, pyrexia of unknown origin, neoplastic and metabolic liver disorders. Usually, a liver biopsy is obtained only after a thorough noninvasive clinical investigation in patients with persistent abnormal liver enzyme elevation. Histopathological evaluation of the liver can provide unavailable information regarding its structure as well as the type and severity of dam- 
age or fibrosis that involve the liver. It is also helpful for monitoring the efficacy of treatment. Percutaneous liver biopsy in children is a procedure with a low rate of major complications and a high rate of minor bleeding as well as no need for intervention and is considered as a safe procedure even in infancy (4-9).

Early diagnosis and prompt treatment offer the only chance of survival, and liver biopsy is the corner stone for the exact diagnosis of liver diseases in children, especially infants (10). Percutaneous liver biopsy is recommended for most infants with neonatal cholestasis, particularly when biliary atresia is highly suspected as a differential diagnosis (11). Percutaneous liver biopsy can be obtained safely in infants and may be helpful in confirming a definitive diagnosis of cholestasis $(11,12)$. The NASPGHAN guideline has recommended that for most infants with undiagnosed cholestasis, a percutaneous liver biopsy should be done (11). Percutaneous liver biopsy can be done as an outpatient procedure. In a study during a 15-year period, 287 inpatient percutaneous liver biopsies were compared with 428 outpatient ones. The authors reported a total complication rate of $6.3 \%$ in the inpatient and $11 \%$ in the outpatient procedures. Only two major complications were reported, but no deaths occurred (13).

The most common histopathological diagnosis in children in this study was chronic hepatitis in $23.1 \%$ of the cases while in a similar study (14) in Iran (capital city of Tehran) an iron overload due to major thalassemia was reported as the most common histological diagnosis (17.5\%). The reason for this difference can be the performance of more pediatric bone marrow transplantations in Tehran compared to Shiraz, and referral of more cases of major thalassemia for liver biopsy as pre-transplant evaluation in Tehran hepatology centers. The frequency of cirrhosis, neonatal hepatitis and familial cholestasis were similar between these two studies in Iran.

In a study in Oman (15), neonatal hepatitis (28.9\%) and biliary atresia (11.8\%) were the most common liver diseases among children, but the frequency of cirrhosis was same as the present study (9.2\% vs. $8.8 \%$ ). These higher rates of neonatal hepatitis and biliary atresia may be due to lower age of the patients in Oman study (15). In a study in Pakistan, Ahmad M. et al. evaluated 100 cases younger than 16 years old, who underwent liver biopsy during a four-year period. They reported secondary hemochromatosis (30\%), biliary atresia (20\%), storage disorders (16\%), cirrhosis (10\%) and neonatal hepatitis (10\%) as the most common histological findings. Chronic hepatitis (6\%), nonspecific reactive hepatitis (3\%) and granulomatous hepatitis (1\%) were considered as the less common diagnoses. They only found one case of hepatoblastoma (1). Also in a study that was conducted in South Africa (16), neonatal hepatitis (19.4\%) and biliary atresia (20.8\%) were reported as the most common histopathological find- ings of liver biopsies in children, but age of the patients in this series was lower than this study. Ramakrishna B. et al. (17) reviewing 134 biopsies obtained from 128 Indian infants and children aged less than 16 years during three years reported a pattern of childhood liver disease. They reported $20.3 \%$ cirrhosis, $17.9 \%$ neonatal hepatitis, and $8.5 \%$ storage disorders as the most common liver biopsy findings. Less common histological findings included Reye's syndrome, fatty liver, granulomas, fulminant hepatitis, chronic active hepatitis, congenital hepatic fibrosis and hepatic malignancies. On that study $23 \%$ of the liver biopsies were non-diagnostic (17).

In a retrospective histopathological study in Nigeria (18), hepatic schistosomiasis (37.5\%) was reported as the most common liver disease in Nigerian children followed by cirrhosis in $25 \%$ and biliary atresia in $4.2 \%$ of the patients. The authors of this study concluded that compared to European countries where neonatal hepatitis and biliary atresia were common, in tropical countries inflection was common because of Schistosoma mansoonsi and Schistosoma hematopoiesis species; but hydatid cyst was found only in $1.9 \%$ of our patients, which is due to better health conditions in Iran (18).

In a 10-year-period study in Pakistan, 48 liver tumors were diagnosed in children less than 18 years. Of these tumors, 39 (81.2\%) were malignant with male to female ratio of 2:1. The authors have reported hepatoblastoma as the most common liver tumor in pediatric age group accounting for $69.2 \%$ ( 27 cases) of all malignant tumors followed by hepatocellular carcinoma that was diagnosed in $15.4 \%$ (six patients) of cases. Other malignant tumors included undifferentiated embryonal sarcoma and biliary rhabdomyosarcoma. They also reported three metastatic tumors during this period (19). The relative high frequency of hepatic malignancies $(6.8 \%)$ in this study is due to the referral of the patients from all over the country to this center as the only pediatric liver transplant center in Iran.

Nonalcoholic fatty liver disease (NAFLD) is one of the leading causes of chronic liver disease in children. The prevalence of NAFLD is increasing and can be predicted by obesity and male sex. It is defined by hepatic fat infiltration in more than $5 \%$ of hepatocytes, in the absence of other causes of liver diseases. NAFLD includes a spectrum of disease beginning from intrahepatic fat accumulation or steatosis. NAFLD is associated with severe metabolic problems and considered as a risk factor for the development of metabolic syndrome. It can progress to fibrosis and cirrhosis and also hepatocellular carcinoma (20). In the present study steatosis was seen in $4.2 \%$ of the patients.

Regarding age, the most common histopathological diagnosis in patients younger than two years old (39.9\% of cases) were metabolic diseases (22.8\%), neonatal hepatitis (17.9\%) and biliary atresia (9.7\%). After two years of age, 
chronic hepatitis became the most common finding especially in 12 - 18-year-old group which was accompanied with more prevalence of autoimmune hepatitis and Wilson disease.

Liver biopsy was not diagnostic in $16.2 \%$ of the patients in the present study which was comparable with Fekade D. study that evaluated the histopathological features of liver disease in hospitalized Ethiopian children (21); but lower than Ramakrishna B. study (17). The relatively-high percentage of non-diagnostic biopsies can be due to lack of more specific tests and enzyme studies on the liver tissue and also absence of electron microscopic examination of liver tissues in our center. Thus, providing the necessary facilities and equipment for special assessments of liver tissue is essential in this center due to the hepatic involvement caused by systemic diseases as well as the important role of the liver biopsy in accurate diagnosis of different liver diseases in children.

\section{Acknowledgements}

The present article was extracted from the thesis written by Zahra Eskandari and Fatemeh Erfanifar and was financially supported by Shiraz University of Medical Sciences, grants No 88-1441.

\section{Authors' Contribution}

Dehghani SM, Haghighat M, Imanieh MH, and Geramizadeh B were involved in the study concept and design, drafting of the manuscript, critical revision of the manuscript, and study supervision; Dehghani SM, Eskandari Z, Erfanifar F, and Malekpour A were in charge of acquisition of data, analysis and interpretation of data, and drafting of the manuscript.

\section{Financial Disclosure}

We have no financial interests related to the materials of the manuscript.

\section{Funding Support}

This work is financially supported by Shiraz University of Medical Sciences, grant No 88-1441.

\section{References}

1. Ahmad M, Afzal S, Roshan E, Mubarik A, Bano S, Khan SA, et al. Usefulness of needle biopsy in the diagnosis of paediatric liver disorders. J Pak Med Assoc. 2005;55(1):24-8.

2. Lai MW, Chang MH, Hsu SC, Hsu HC, Su CT, Kao CL, et al. Differ- ential diagnosis of extrahepatic biliary atresia from neonatal hepatitis: a prospective study. J Pediatr Gastroenterol Nutr. 1994;18(2):121-7.

3. Dehghani SM, Haghighat M, Imanieh MH, Geramizadeh B. Comparison of different diagnostic methods in infants with Cholestasis. World J Gastroenterol. 2006;12(36):5893-6.

4. Holtz T, Moseley RH, Scheiman JM. Liver biopsy in fever of unknown origin. A reappraisal. J Clin Gastroenterol. 1993;17(1):29-32.

5. Ozawa K, Mori K, Morimoto T. Evaluation of hepatic function. Curr Opin Gen Surg. 1994:17-23.

6. Westheim BH, Ostensen AB, Aagenaes I, Sanengen T, Almaas R. Evaluation of risk factors for bleeding after liver biopsy in children. J Pediatr Gastroenterol Nutr. 2012;55(1):82-7.

7. El-Shabrawi MH, El-Karaksy HM, Okahsa SH, Kamal NM, El-Batran G, Badr KA. Outpatient blind percutaneous liver biopsy in infants and children: is it safe? Saudi J Gastroenterol. 2012;18(1):26-33.

8. Matos H, Noruegas MJ, Goncalves I, Sanches C. Effectiveness and safety of ultrasound-guided percutaneous liver biopsy in children. Pediatr Radiol. 2012;42(11):1322-5.

9. Potter C, Hogan MJ, Henry-Kendjorsky K, Balint J, Barnard JA. Safety of pediatric percutaneous liver biopsy performed by interventional radiologists. J Pediatr Gastroenterol Nutr. 2011;53(2):202-6.

10. Bezerra JA, Balistreri WF. Cholestatic syndromes of infancy and childhood. Semin Gastrointest Dis. 2001;12(2):54-65.

11. Moyer V, Freese DK, Whitington PF, Olson AD, Brewer F, Collett $\mathrm{RB}$, et al. Guideline for the evaluation of cholestatic jaundice in infants: recommendations of the North American Society for Pediatric Gastroenterology, Hepatology and Nutrition. J Pediatr Gastroenterol Nutr. 2004;39(2):115-28.

12. Fox VL, Cohen MB, Whitington PF, Colletti RB. Outpatient liver biopsy in children: a medical position statement of the North American Society for Pediatric Gastroenterology and Nutrition. J Pediatr Gastroenterol Nutr. 1996;23(3):213-6.

13. Weigand K. Percutaneous liver biopsy: retrospective study over 15 years comparing 287 inpatients with 428 outpatients. J Gastroenterol Hepatol. 2009;24(5):792-9.

14. Monajemzadeh M, Tabriz HM, Mahjoub F, Fallahi G, Farahmand F. Liver needle biopsy in Iraninan pediatric patients: diagnostic significance and pattern of liver diseases. Indian J Pathol Microbiol. 2009;52(1):10-3.

15. Akinbami FO, Venugopalan P, Nirmala V, Suresh J, Abiodun P. Pattern of chronic liver disease in Omani children-a clinicopathological review. West Afr J Med. 2004;23(2):162-6.

16. Muthuphei MN. Childhood liver diseases in Ga-Rankuwa Hospital, South Africa. East Afr Med J. 2000;77(9):508-9.

17. Ramakrishna B, Date A, Kirubakaran C, Raghupathy P. The pat tern of liver disease in Indian children: a review of 128 biopsied cases. Ann Trop Paediatr. 1993;13(2):159-63.

18. Obafunwa JO, Elesha SO. Childhood liver diseases in Jos, Nigeria: a retrospective histopathological study. East Afr Med J. 1991;68(9):702-6

19. Zaman S, Hanif G, Hussain M, Basit Z, Khan S, Rathore Z, et al Hepatic tumours in childhood: an experience at the Children Hospital and Institute of Child Health, Lahore. J Pak Med Assoc 2011;61(11):1079-82.

20. Giorgio V, Prono F, Graziano F, Nobili V. Pediatric non alcoholic fatty liver disease: old and new concepts on development, progression, metabolic insight and potential treatment targets. BMC Pediatr. 2013;13:40.

21. Fekade D. Histopathological features of liver disease in hospitalized Ethiopian patients. Ethiop Med J.1989;27(1):9-13. 